\title{
Early Detection of Fetal Malformation, a Long Distance Yet to Cover! Present Status and Potential of First Trimester Ultrasonography in Detection of Fetal Congenital Malformation in a Developing Country: Experience at a Tertiary Care Centre in India
}

\author{
Namrata Kashyap, Mandakini Pradhan, Neeta Singh, and Sangeeta Yadav \\ Department of Maternal and Reproductive Health, Sanjay Gandhi Post Graduate Institute of Medical Sciences (SGPGIMS),
} Lucknow 226 014, India

Correspondence should be addressed to Namrata Kashyap; dr.nmrata@gmail.com

Received 4 September 2015; Accepted 27 October 2015

Academic Editor: R. L. Deter

Copyright (C) 2015 Namrata Kashyap et al. This is an open access article distributed under the Creative Commons Attribution License, which permits unrestricted use, distribution, and reproduction in any medium, provided the original work is properly cited.

\begin{abstract}
Background. Early detection of malformation is tremendously improved with improvement in imaging technology. Yet in a developing country like India majority of pregnant women are not privileged to get timely diagnosis. Aims and Objectives. To assess the present status and potential of first trimester ultrasonography in detection of fetal congenital structural malformations. Methodology. This was a retrospective observational study conducted at Sanjay Gandhi Postgraduate Institute of Medical Sciences. All pregnant women had anomaly scan and women with fetal structural malformations were included. Results. Out of 4080 pregnant women undergoing ultrasound, 312 (7.6\%) had fetal structural malformation. Out of 139 patients who were diagnosed after 20 weeks, $47(33.8 \%)$ had fetal structural anomalies which could have been diagnosed before 12 weeks and 92 (66.1\%) had fetal malformations which could have been diagnosed between 12 and 20 weeks. Conclusion. The first trimester ultrasonography could have identified $50 \%$ of major structural defects compared to $1.6 \%$ in the present scenario. This focuses on the immense need of the hour to gear up for early diagnosis and timely intervention in the field of prenatal detection of congenital malformation.
\end{abstract}

\section{Introduction}

Fetal structural malformations are seen in 3 to $5 \%$ of all pregnancies [1]. Detection of malformation is tremendously improved with improvement in imaging technology. In majority of countries worldwide, second trimester scan between 18 and 22 weeks remains the standard of care for fetal anatomical assessment; however, most recent literature shows a significant improvement in detection of fetal abnormalities in first trimester of pregnancy [2]. Besides nuchal abnormalities a wide range of central nervous system, heart, anterior abdominal wall, urinary tract, and skeletal abnormalities can be diagnosed between 11 and 14 weeks of scan. The clear benefits of first trimester ultrasound are early detection and exclusion of major congenital anomalies (not compatible with life or followed by severe handicap), reassurance, and relatively easier pregnancy termination if required.

Currently, the review of recent literature suggests classification of fetal abnormalities as always detectable, potentially detectable, and undetectable till first trimester and anomaly scan. The diagnostic efficacy of first trimester anomaly scan and echocardiography between 11 and 14 weeks has been assessed in medium risk population by Becker and Wegner [3]. The prevalence of major anomalies in their study group was $2.8 \%$. The overall detection rate of fetal anomalies including cardiac defects was $84 \%$ and increased with raised nuchal thickness particularly more than $2.5 \mathrm{~mm}$. This highlights the scope of first trimester scan apart from its conventional role in detection of chromosomal abnormality.

First trimester screening is now no more limited to detection of raised nuchal thickness (NT). Becker [4] et al. 
analysed 6879 cases to assess the prevalence and detection rate of major anomalies by applying first trimester anomaly scan and fetal echocardiography. They concluded that a significant number of fetal anomalies occur with normal NT and more than half of them could be detected in first trimester. Hence, even fetuses with normal NT should be offered first trimester anomaly scan and fetal echocardiography considering the ethical principles of nonmaleficence, justice, and respect for autonomy of pregnant women. Even in this era the benefits of this established technology are not in the reach of all. A vast majority of patients in India are not yet undergoing anomaly scan. We frequently encounter malformations always or potentially detectable during first trimester scan at third trimester or in postnatal period. It depends on both the expertise and resources available along with the awareness and sensitization in general population. This fact of diagnosis is particularly more important in countries like India where medical termination of pregnancy [5] is legally allowed up to 20 weeks of gestation irrespective of malformation being lethal. We see a fair number of patients who are diagnosed with fetal malformation beyond 20 weeks and in that situation they are forced to seek termination services at small substandard centres since they get refusals from all relatively good hospitals due to legal issues associated with termination. Many of such patients get deteriorated due to septic abortion and unnecessary hysterotomy and so forth. Question then arises that where lies the fault, the awareness of the patients or the expertise of the sonologist.

Henceforth, the study was planned to assess the prevalence of fetal malformation in a tertiary care referral centre and to assess the present status of first trimester ultrasonography in the detection of fetal malformations in a tertiary care centre in India.

\section{Materials and Methods}

This was a retrospective observational study conducted at Sanjay Gandhi Postgraduate Institute of Medical Sciences. All pregnant women attending Department of Maternal and Reproductive Health, OPD, from August 2009 till October 2013 were enrolled in the study. All pregnant women underwent ultrasound (General electrical Voluson S8) and those with fetal structural malformations were evaluated. Malformations were classified according to gestational age of diagnosis, system involved, and type of malformation. Descriptive proportions and frequencies have been used to depict the data.

\section{Results}

A total number of 4080 pregnant women underwent USG and amongst them $312(7.6 \%)$ patients had fetal structural malformation. The malformations were classified according to gestational age as shown in Table 1. Malformations were classified according to various systems as shown in Table 2.

3.1. Malformations Detected prior to 20 Weeks. Out of total malformed fetuses, 103 (33\%) were detected prior to 20 weeks of gestational age and $209(66.9 \%)$ were detected after
TABLE 1: Number of malformations at different gestational age.

\begin{tabular}{lc}
\hline Gestational age & Number \\
\hline$<12$ weeks & 5 \\
$12-20$ weeks & 98 \\
$>20$ weeks & 209 \\
\hline
\end{tabular}

20 weeks of gestational age. Out of 103 women who were diagnosed with fetal malformations before 20 weeks, only 5 $(1.6 \%)$ were detected prior to 12 weeks of gestational age and the remaining 98 (31.4\%) were diagnosed between 12 and 20 weeks. Six patients amongst them presented before 12 weeks but malformations were missed and diagnosed later between 12 and 20 weeks. These cases were omphalocele, osteogenesis imperfecta, harlequin ichthyosis, Stickler syndrome, Fraser syndrome, and Dandy-Walker malformation. These conditions, however, are known to present late.

Out of 103 patients diagnosed to have malformation prior to 20 weeks, 80 patients willingly underwent termination of pregnancy in view of malformation being lethal like a fetus with occipital encephalocoele terminated at 20 weeks of gestational age (Figure 1). We had prescribed protocol of oral mifepristone $(200 \mathrm{mg})$ followed by misoprostol induction after 48 hours of mifepristone. Three patients were lost to follow-up. Ten patients had nonlethal malformation and were willing to continue pregnancy. All of them had postpartum neonatal intervention in the Department of Pediatric Surgery, Neonatology and Plastic Surgery, respectively (for posterior urethral valve, extra lobar sequestration, tracheoesophageal fistula, anorectal malformation, congenital diaphragmatic hernia with good LH ratio, meningocele, polycystic kidneys, megacystis, vesicoureteral reflux, and cleft lip palate).

Ten patients refused to continue pregnancy despite malformation being lethal. They had obstetrical procedure at their convenient places. Four amongst them had preterm still birth and six babies died in neonatal period. Biggest agony is that two amongst those continuing pregnancies with known lethal malformations had hysterotomy and two had cesarean section for anomalous fetus which could have been avoided.

We found that with the present available technology majority of malformation could be diagnosed before 20 weeks (Box 1).

First trimester sonography has huge potential of diagnosing fetal anomalies. We found that there are few malformations which could be easily diagnosed before 12 weeks (Box 2).

Five patients were diagnosed prior to 12 weeks for neural tube defect, holoprosencephaly, gastroschisis, cystic hygroma, and anencephaly. All of them had easy termination of pregnancy.

3.2. Malformations Detected after 20 Weeks. Out of 312 pregnant women with malformations, 209 (66.9\%) were diagnosed after 20 weeks. 109 had their first USG after 20 weeks and 100 had USG prior to 20 weeks but malformations were missed.

Out of those 100 patients, 6 patients presented to our institute before 20 weeks and malformations were not confirmed 
TABLE 2: Classification of malformation according to the system involved.

\begin{tabular}{|c|c|c|c|}
\hline CNS, brain & Number & Skeletal & Number \\
\hline Neural tube defects & 30 & Achondroplasia & 2 \\
\hline Porencephaly & 1 & Hypochondroplasia & 2 \\
\hline Anencephaly & 11 & Osteogenesis imperfecta & 3 \\
\hline Occipital encephalocoele & 8 & Short rib polydactyly & 6 \\
\hline Iniencephaly & 2 & Thanatophoric dysplasia & 1 \\
\hline Ventriculomegaly & 14 & Single forearm bone & 1 \\
\hline Arachnoid cyst & 2 & Cooks syndrome & 1 \\
\hline Holoprosencephaly & 8 & Respiratory & \\
\hline Agenesis of corpus callosum & 8 & CCAM & 7 \\
\hline Dandy-Walker malformation & 16 & Pleural effusion & 1 \\
\hline Diastematomyelia & 2 & Congenital high airway obstruction & 3 \\
\hline Vermian agenesis & 2 & Extralobar pulmonary sequestration & 2 \\
\hline Genitourinary & & Heart & \\
\hline ADPKD & 3 & Structural cardiac malformations & 44 \\
\hline ARPKD & 1 & Congenital heart blocks & 8 \\
\hline Megacystis & 1 & Pericardial effusion & 2 \\
\hline Gonadal cyst & 2 & Structural and rhythmic & 1 \\
\hline Hydronephrosis & 12 & AV malformation & \\
\hline Lower urinary tract obstruction & 9 & Vein of Galen malformation & 1 \\
\hline Horseshoe kidney & 1 & Klippel-Trenaunay-Weber syndrome & 1 \\
\hline Unilateral multicystic kidney & 17 & Others & \\
\hline Bilateral cystic kidney disease & 9 & Fetal goitre & 1 \\
\hline Gastrointestinal & & Cystic hygroma & 6 \\
\hline Fetal ascites & 1 & Multiple & \\
\hline Meconium peritonitis & 2 & Limb body wall complex & 2 \\
\hline Gastroschisis & 3 & VACTERL & 1 \\
\hline Mesenteric cyst & 1 & Meckel-Gruber syndrome & 6 \\
\hline Enteric duplication cyst & 1 & Multiple malformations & 20 \\
\hline Hirschsprung disease & 1 & Fetal tumors & \\
\hline Tracheoesophageal fistula & 2 & Adrenal neuroblastoma & 1 \\
\hline Congenital diaphragmatic hernia & 3 & Teratoma & 3 \\
\hline Duodenal atresia & 4 & Sacrococcygeal teratoma & 1 \\
\hline Isolated fetal ascites & 2 & & \\
\hline Omphalocele & 6 & & \\
\hline
\end{tabular}

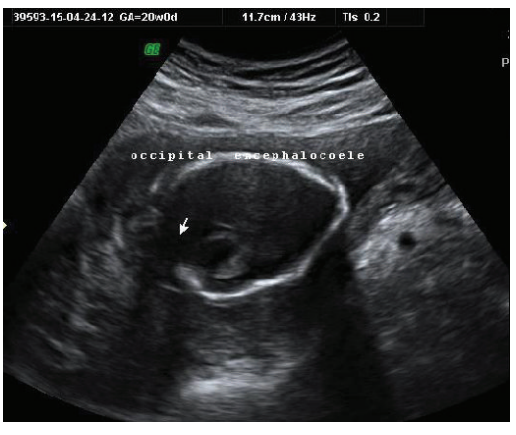

(a)

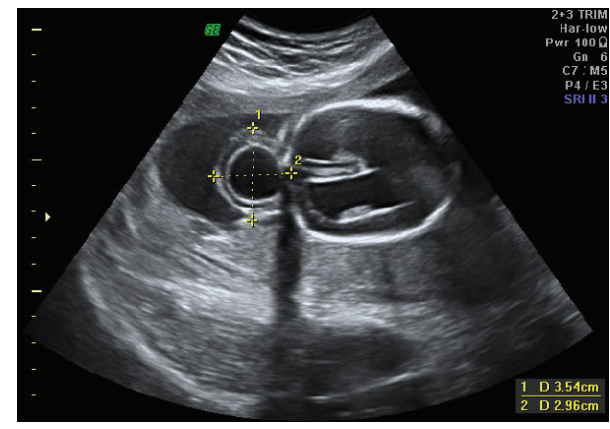

(b)

Figure 1: Occipital encephalocoele diagnosed at 20 weeks which was terminated. 
Skeletal dysplasia (achondrogenesis, thantophoric dysplasia, osteogenesis imperfecta), frequently continued as wrong dates Limb body wall complex

Renal agenesis, frequently continued as severeoligohydramnious

Agenesis of corpus callosum,

Occipital encephalocoele

Porencephaly, Posterior fossa cyst

Spinal defects like diastematomelia, hemivertebrae, iniencephaly

Ventriculomegaly

Multiple malformations (Meckel grcuber, VACTREL, Kleipell Trenaunay Weber, Cooks)

Hydronephrosis, VUR, PUJ Obstruction

Congenital Diaphragmatic Hernia

Congenital Cystic Adenomatoid Malformation

Hypoplastic left and right heart

Lower urinary tract obsruction

Bilateral multicystic dysplastic kidney

Megacystis

Tracheoesophageal fistula

Meconium peritonitis

Congenital high airway obstruction

Urorectal septal malformations

Teratomas,

Isolated pleural effusion and

Extralobar pulmonary sequestration

TRAP sequence

Cystic hygroma

Box 1: List of common malformations detected before 20 weeks.

Acrania, anencephaly, encephaocoele, etopia cordis (100\% detectable)

Spina bifida, hydrocephalous, holoprosencephaly

Cystic hygroma,

Hypoplastic left heart syndrome, atrioventricular septal defect

Limb reduction defects

Megacystis

Skeletal dysplasia

Box 2: List of common malformations we found usually detectable before 12 weeks.

until 24 weeks. In 94 women, they went for USG prior to 20 weeks at some other centre and malformation was missed. Amongst those six patients who presented to SGPGI prior to 20 weeks but were missed, there were one case each of Dandy-Walker malformation, autosomal dominant polycystic kidneys, late onset hydrocephalus, and tetralogy of Fallot. All of these tend to be diagnosed late. Two fetuses, one with cleft lip and one with neural tube defect, could have been diagnosed but were missed. There exists a group of malformation which lies in the grey zone of diagnosis before 20 weeks (Box 3).

Out of 209 detected cases after 20 weeks, 70 (33.4\%) patients had malformations which were detected after 20 weeks and are acceptable because these include conditions which tend to be diagnosed late in gestation like hydrocephalus (Figures 2(a) and 2(b)), agenesis of corpus callosum (Figure 2(c)), congenital cystic adenomatoid malformation (Figure $2(\mathrm{~d})$ ), various cardiac structural malformations, cystic kidney diseases, horseshoe kidney, DandyWalker malformations and variants, vein of Galen aneurysm, duodenal atresia, fetal goitre, intra-abdominal tumours, gonadal cyst, Hirschsprung disease, and isolated fetal ascites.

3.3. Malformation Missed. Even though missed in first trimester, in 139 (66.5\%) patients, fetal malformations could have been diagnosed between 12 and 20 weeks as shown in Box 1. These included malformations like neural tube defect (Figures 3(a) and 3(b)), acrania-exencephaly-anencephaly sequence (Figures 4(a), 4(b), and 4(c)), skeletal dysplasia (Figures 5(a), 5(b), and 5(c)), multicystic dysplastic kidneys (Figure 5(d)), and limb body wall complex (Figures 6(a), 6(b), and $6(\mathrm{c}))$.

Prenatal interventions in very unique complications of monochorionic twins have become the treatment of choice [6] but diagnosis of acardiac twinning was delayed till 24 weeks (Figure 6(d)). This delayed pick-up of these potentially salvageable conditions leads to high likelihood of adverse pregnancy outcome. The patient had demise of normal cotwin also at 28 weeks. 
Agenesis of corpus callosum

Cystic congenital adenomatoid malformation, extralobar sequestration

Dandywalker malformation and variants

Duodenal atresia

Hydronephrosis, renal agenesis, duplex kidney

Bowel obstruction

Box 3: List of malformations we found undetectable before 12 weeks and difficult between 12-20 weeks.

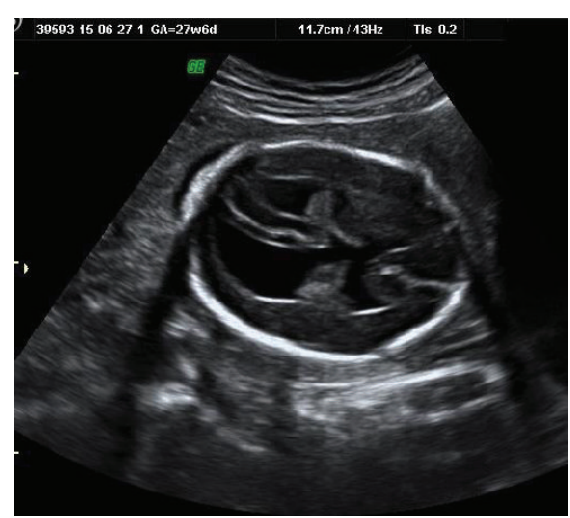

(a)

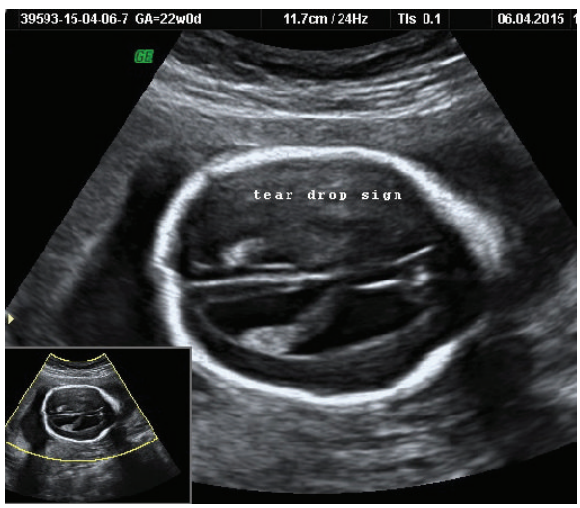

(c)

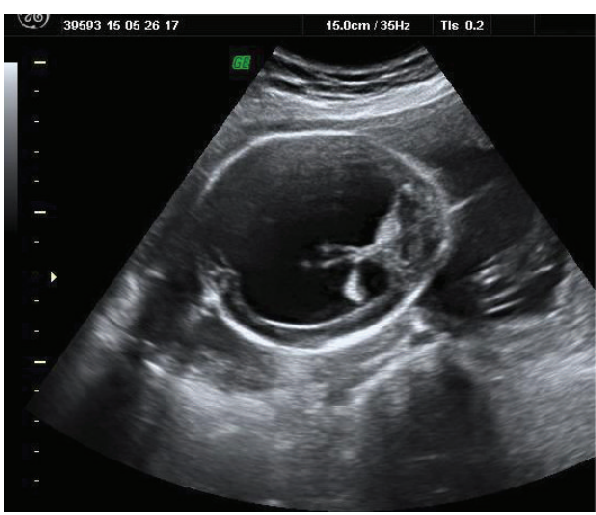

(b)

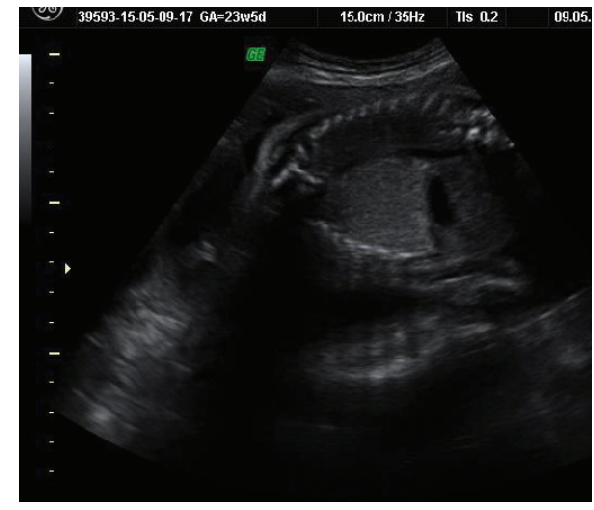

(d)

Figure 2: (a and b) show late onset hydrocephalus diagnosed at 27 weeks and 32 weeks, noted to occur late as a part of MASA syndrome (patient had history of X-linked hydrocephalus). (c) shows agenesis of corpus callosum which is known to be said with confirmation at later gestation. (d) shows fetal micro cystic congenital cystic adenomatoid malformation (CCAM) which is notorious to be missed early.

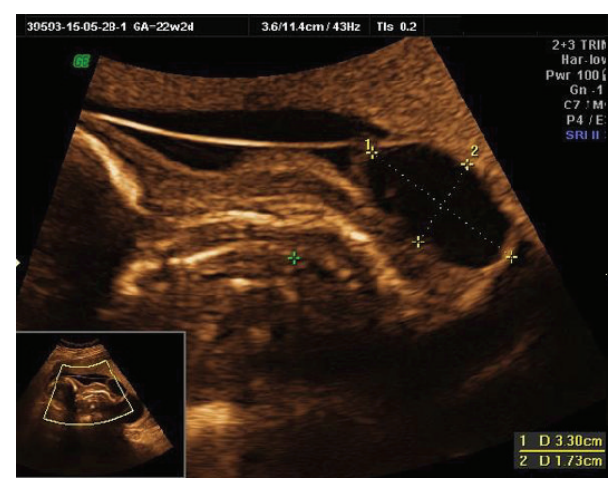

(a)

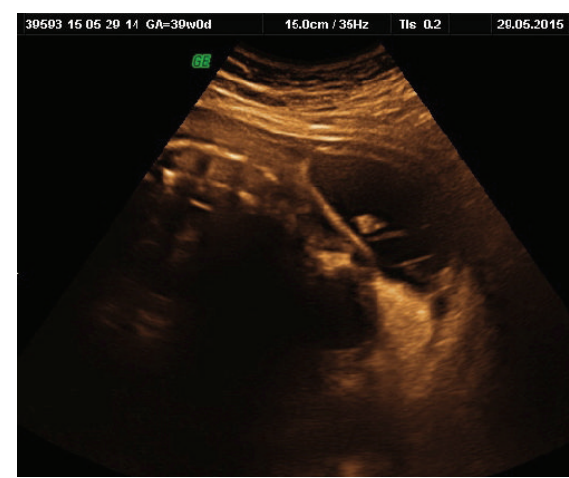

(b)

FIGURE 3: ( $\mathrm{a}$ and $\mathrm{b}$ ) show two cases of neural tube defect diagnosed at advanced gestational ages of 22 weeks and 39 weeks. 


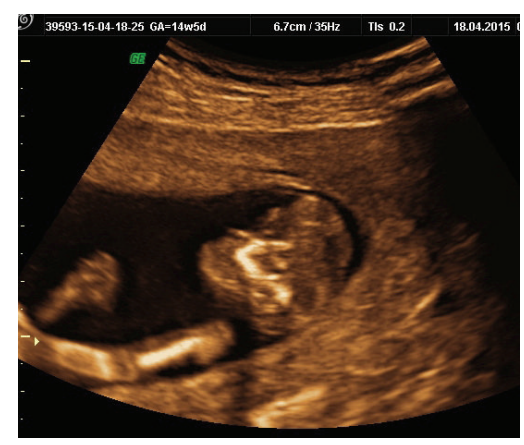

(a)

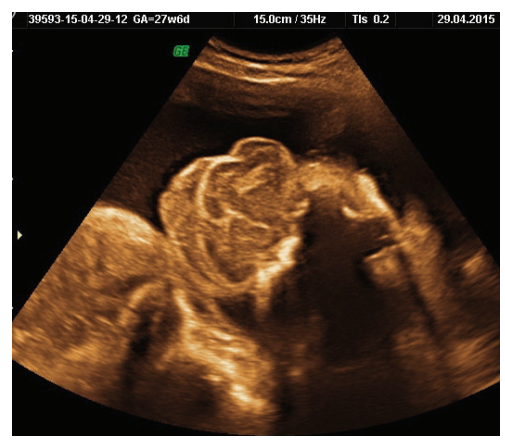

(b)

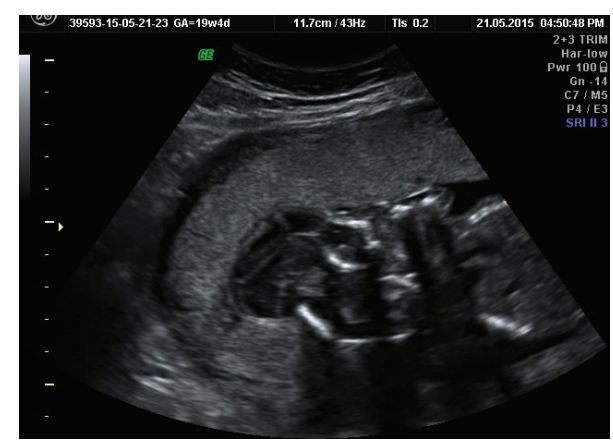

(c)

Figure 4: (a, b, and c) show fetal exencephaly that could be diagnosed at 14 weeks but was missed and diagnosed at 19 weeks and as late as 27 weeks.

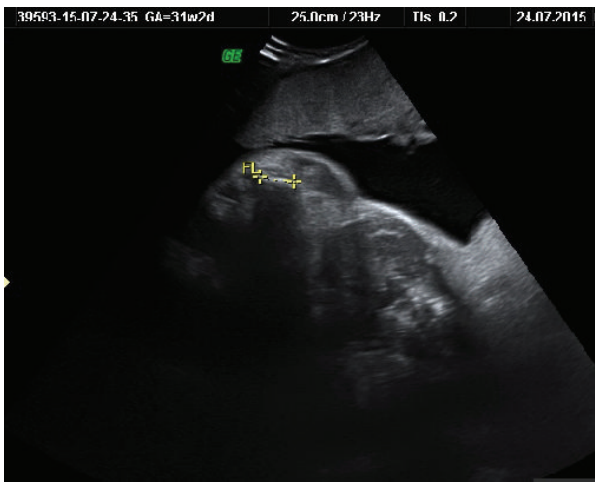

(a)

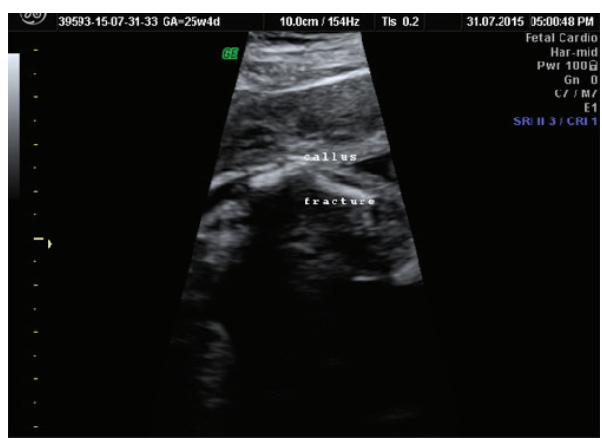

(c)

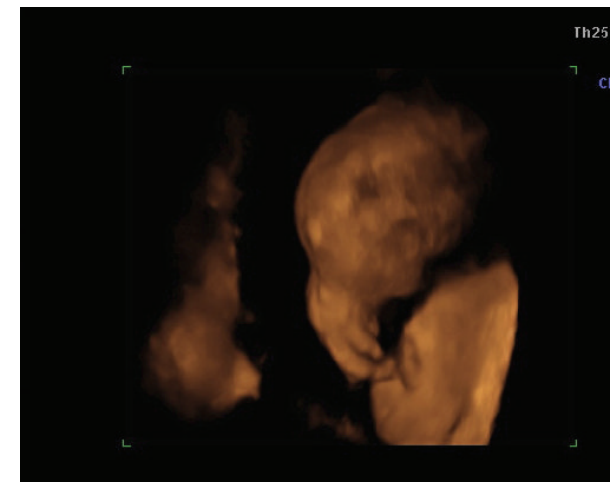

(b)

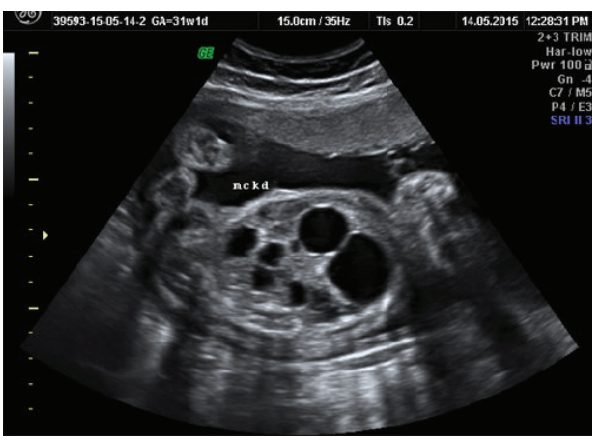

(d)

FIgURE 5: (a, b, and c) show skeletal malformation achondrogenesis (3D), thanatophoric dysplasia, and osteogenesis imperfecta. All of them potentially diagnosable before 12 weeks and usually before 20 weeks were missed and were diagnosed late. (d) shows bilateral multicystic dysplastic kidney diagnosed at 31 weeks, with fetus being continued as oligohydramnios.

\section{Discussion}

The overall prevalence of severe and lethal fetal structural malformation in our study was $7.6 \%$ which was higher than that reported in the literature for general population (3$5 \%$ ), possibly because it was a referral centre for high risk pregnancy and fetal medicine; there is overreporting of cases. Our study calculated that CNS malformations were most common in our study population. As such, preconceptional folic acid is not commonly practiced in our study population. We realize that almost half $(52.1 \%)$ of our patients had their first USG for anomaly detection after 20 weeks. It reflects the existing darkness of unawareness and vacuum of knowledge in patients and also in basic health care that are first to encounter pregnant women.

We found that, out of the total number of women with diagnosed fetal malformation, 203 (65\%) presented before 20 weeks. Hence, equally important is the fact to realize that almost half of these patients who had malformations detected after 20 weeks had their obstetrical sonography before 20 weeks and were missed. This missing out of an anomaly may be because of scarcity of good resolution 


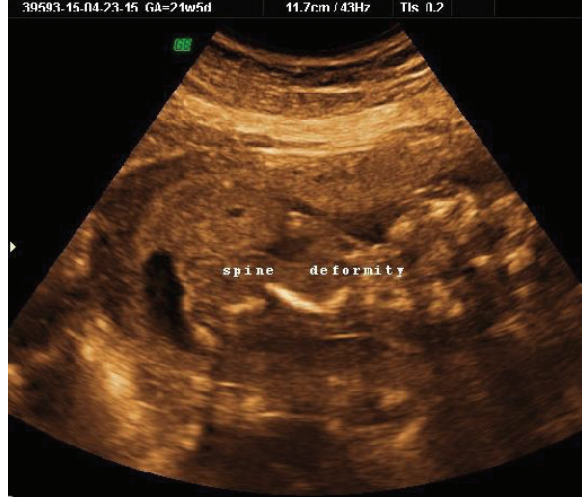

(a)

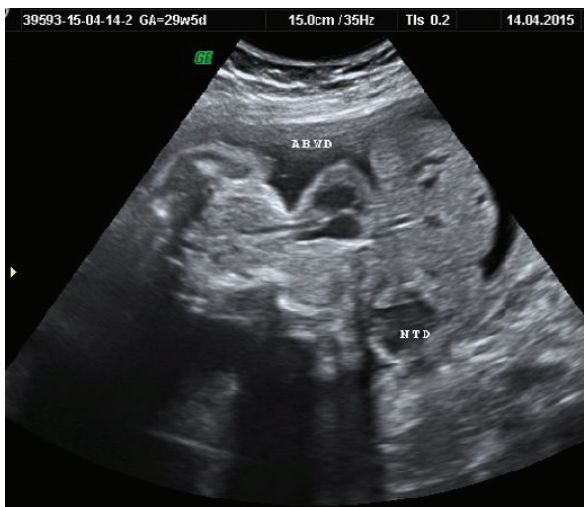

(c)

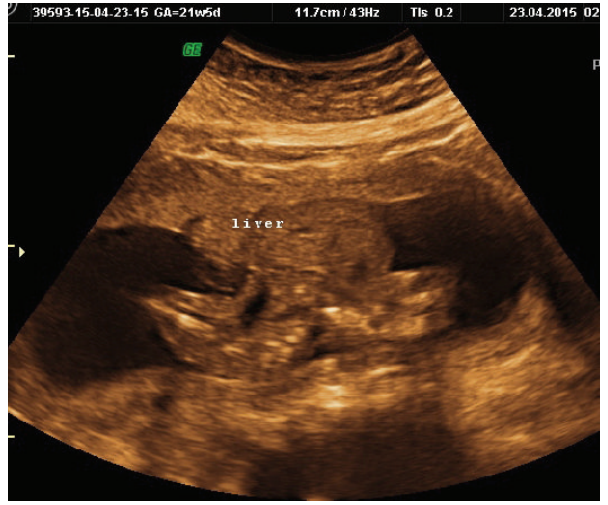

(b)

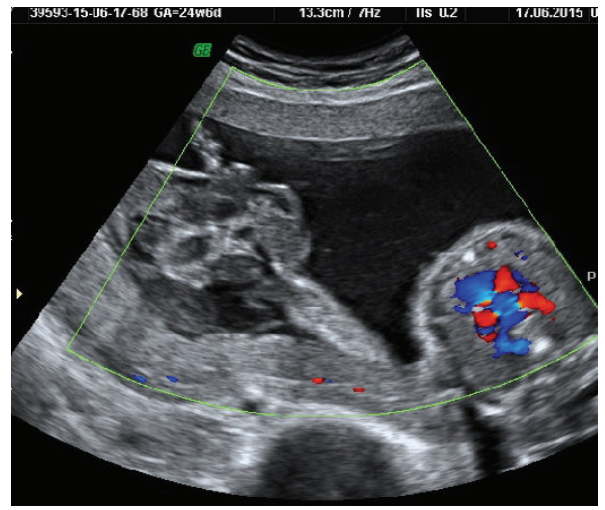

(d)

FIGURE 6: (a, b, and c) show gross fetal deformity limb body wall complex, diagnosis delayed till 21 weeks in one fetus (Figures 6(a) and 6(b)) and 29 weeks in other fetuses (Figure 6(c)). (d) shows acardiac twin (twin reverse arterial perfusion sequence) diagnosed at 25 weeks when the normal twin had already decompensated making any intervention difficult.

machines, busy schedules, and lack of expertise as well. For years together, there have been substantial advances in magnification imaging and signal processing which increased the ability to visualize fetal anatomy; there has been great concern on the possibility to diagnose a wide range of fetal anomalies at the time of nuchal translucency scan by transvaginal and transabdominal sonography [7-9].

Almost half of malformations in our study were amenable to be diagnosed in first trimester as reported in current literature. These fetuses were having malformations like neural tube defects, anencephaly, holoprosencephaly, and gastroschisis (Box 2). Castro-Aragon and Levine [10] reported that $60-67 \%$ of malformations could have been diagnosed prior to 12 weeks. This is far away from our scenario where we found that only $1.6 \%(5 / 312)$ were diagnosed prior to 12 weeks. This is possibly due to the lack of awareness and lack of expertise as well. Fong et al. [11] in their study scanned 8,537 women between 11 and 14 weeks of gestation (crown rump length, 45$84 \mathrm{~mm}$ ); there were 175 fetuses with an increased NT. Besides nuchal abnormalities, a wide range of other congenital anomalies can be diagnosed with US at 11-14 weeks of gestation, including defects of the central nervous system, heart, anterior abdominal wall, urinary tract, and skeleton. Oztekin et al. [12] analyzed 1085 pregnancies; 21 (1.93\%) fetuses had at least one major structural defect considered detectable by routine ultrasound screening. $14(1.29 \%)$ were identified at early (first trimester) screening and an additional $5(0.47 \%)$ were identified at late (second trimester) USG. They found that majority of fetal structural abnormalities can be detected by sonographic screening at 11-14 weeks, but detailed fetal anatomic survey performed at 18-22 weeks should not be abandoned.

Rossi and Prefumo [13] also laid stress that first trimester ultrasound can detect half of fetal malformations. They included nineteen studies on 78,002 fetuses, with 996 with malformations that were confirmed by postnatal or postmortem examinations. USG at 11 to 14 weeks detected malformation in 472 of the malformed fetuses (51\%). Detection rate was highest for neck anomalies (92\%) and lowest for limbs, face, and genitourinary anomalies (34\% for each). The presence of associated anomalies appears to increase the accuracy of early ultrasonography. Multiple defects were more likely to be identified than isolated malformations $(60 \%$ versus $44 \%$ ). Detection rates ranged from $1 \%$ to $49 \%$ for spina bifida or hydrocephalus, ranged from $50 \%$ to $99 \%$ for valvular disease and septal defects, were $100 \%$ for acrania and anencephaly, and were $0 \%$ for corpus callosum agenesis and bladder exstrophy. Combination of transabdominal and 
transvaginal techniques resulted in a $62 \%$ detection rate versus $51 \%$ for transabdominal technique only and $34 \%$ for transvaginal technique only.

Although first trimester ultrasound can detect about 50\% of fetal malformations, it cannot replace second trimester ultrasound because several malformations develop later than the first trimester. Also to be kept in mind is the fact that accuracy of early ultrasonography can be compromised by transient findings like midgut herniation, small septal defects, and hydronephrosis which might get resolved during intrauterine life.

Iliescu et al. [14] did a prospective two-centre 2-year study of 5472 consecutive unselected pregnant women examined at 12 to $13+6$ gestational weeks. The first trimester scan identified $40.6 \%$ of the cases detected overall and $76.3 \%$ of major structural defects. Major congenital heart disease (either isolated or associated with extracardiac abnormalities) was 90\%. Major central nervous system anomalies were $69.5 \%$. Fetuses with increased nuchal translucency (NT), the first trimester DR for major anomalies, were $96 \%$ compared to $66.7 \%$ amongst those with normal NT.

There have been several studies seeing application for an extended protocol in which first trimester sonography is supported by a second anomaly scan. The obvious advantage of an extended protocol is that parents are offered the option of earlier and safer termination of pregnancy for the large majority of severe/lethal abnormalities.

Early ultrasound might be more accurate than second trimester ultrasonography for detection of malformations associated with oligohydramnios and anhydramnios which lead to poor visualization at later gestation necessitating amnioinfusion.

We have applied this kind of protocol at our centre particularly in high risk women. First trimester sonography with targeted imaging for fetal malformation appeared particularly more helpful in high risk women with previous history of fetus/neonates with malformations, known risk factors, for example, type 2 diabetes, patients prone for teratogenicity, for example, thromboembolic and valve replacement patients on warfarin, methotrexate intake for connective tissue disorders, and so forth, antiepileptic, chemotherapeutic drugs, and history of infection exposure like rubella. We diagnosed and terminated patients before 16 weeks with rubella exposure and subsequent pulmonary stenosis, severe bony stippling and craniofacial malformation associated with high doses warfarin, VSD associated with type 2 diabetes, neural tube defect with antiepileptic and tetraphocomelia with chemotherapeutic agents, renal agenesis in previous history of Fraser's syndrome, encephalocoele in previous Meckel-Gruber syndrome, ARPKD and ADPKD, and so forth.

However, a detailed first trimester examination protocol involves supplementary resources: additional examination time and specialized personnel for the abnormal suspected/detected cases. Healthcare systems are yet to determine whether early first trimester diagnosis of most major structural abnormalities is cost-effective. Previous research, albeit using inferior ultrasound technology and a less extended protocol, found that the first trimester anomaly scan was cost-efficient in terms of medical and economic expenses, although they obtained lower detection rates [15, 16].

The present research about the effectiveness of early ultrasonography in the diagnosis of structural defects does have some conflicts, which made it a challenge that to what extent structural congenital abnormalities could be detected by the routine scanning of fetal anatomy combined with nuchal translucency measurement [17]. Few other basic prerequisites associated with early prenatal diagnosis consist of the high experience required and high costs in terms of time and equipment [18]. Even with all these circumstances, the situation in our country is such that a huge number of patients, 209 (66.9\%), were diagnosed after 20 weeks which shows the lacunae which need to be filled.

\section{Conclusion}

In our study we realized that even in a tertiary care centre only $1.6 \%$ fetuses with malformation are identified in first trimester. In a way, it throws light on the importance of screening as well as an immense need for early diagnosis and timely intervention in the field of prenatal detection of congenital malformation.

A detailed examination of fetal anatomy during the routine 11-14 weeks of gestation scan provides a comprehensive assessment of fetal anatomy and can detect approximately half of major structural defects in both low-risk and highrisk pregnancies. Detection rate increases markedly beyond 13 weeks of gestation compared with 11 weeks of gestation. We have seen to be better convinced to diagnose holoprosencephaly, achondrogenesis, osteogenesis imperfecta, and spondylocostal dysostosis at 14 weeks compared to 12 weeks. It is also expected that because of the late development of some organ systems and the delayed onset of a significant number of major anomalies in the second and third trimester it is very unlikely that the early scan may replace second trimester ultrasonography.

We need to identify structural malformations before 20 weeks except those conditions which are said to appear further late or reported with confirmation at a later gestational age like few posterior fossa abnormalities, duodenal atresia, and few renal abnormalities. The most important implication is safe termination and avoiding maternal threat to life by forced termination at resourceless and substandard centres. There could be an option of incorporating anomaly scan between 18 and 20 weeks in our health plans and guides at well registered centres with expertise at reasonable cost.

Focus and emphasis should aim at detection of malformation earlier than 12 weeks owing to the very unique and clear facts that first trimester detection leads to easy termination of pregnancy and lessening of women's mental, physical, and psychological trauma.

\section{Conflict of Interests}

The authors declare that there is no conflict of interests regarding the publication of this paper. 


\section{References}

[1] E. Garne, H. Dolk, M. Loane, and P. A. Boyd, "EUROCAT website data on prenatal detection rates of congenital anomalies," Journal of Medical Screening, vol. 17, no. 2, pp. 97-98, 2010.

[2] A. Syngelaki, T. Chelemen, T. Dagklis, L. Allan, and K. H. Nicolaides, "Challenges in the diagnosis of fetal non-chromosomal abnormalities at 11-13 weeks," Prenatal Diagnosis, vol. 31, no. 1, pp. 90-102, 2011.

[3] R. Becker and R.-D. Wegner, "Detailed screening for fetal anomalies and cardiac defects at the 11-13-week scan," Ultrasound in Obstetrics and Gynecology, vol. 27, no. 6, pp. 613-618, 2006.

[4] R. Becker, L. Schmitz, S. Kilavuz, M. Stumm, R.-D. Wegner, and U. Bittner, "Normal' nuchal translucency: a justification to refrain from detailed scan? Analysis of 6858 cases with special reference to ethical aspects," Prenatal Diagnosis, vol. 32, no. 6, pp. 550-556, 2012.

[5] Medical termination of pregnancy act, 2002, Ministry of Health and Family Welfare, Government of India, http://mohfw .nic.in/index1.php?lang=1\&level=3\&sublinkid=3613\&lid=2597.

[6] G. Pagani, F. D’Antonio, A. Khalil, A. Papageorghiou, A. Bhide, and B. Thilaganathan, "Intrafetal laser treatment for twin reversed arterial perfusion sequence: cohort study and metaanalysis," Ultrasound in Obstetrics and Gynecology, vol. 42, no. 1, pp. 6-14, 2013.

[7] A. P. Souka and K. H. Nicolaides, "Diagnosis of fetal abnormalities at the 10-14-week scan," Ultrasound in Obstetrics and Gynecology, vol. 10, no. 6, pp. 429-442, 1997.

[8] L. Dugoff, "Ultrasound diagnosis of structural abnormalities in the first trimester," Prenatal Diagnosis, vol. 22, no. 4, pp. 316320,2002 .

[9] B. J. Whitlow and D. L. Economides, "The optimal gestational age to examine fetal anatomy and measure nuchal translucency in the first trimester," Ultrasound in Obstetrics and Gynecology, vol. 11, no. 4, pp. 258-261, 1998.

[10] I. Castro-Aragon and D. Levine, "Ultrasound detection of first trimester malformations: a pictorial essay," Radiologic Clinics of North America, vol. 41, no. 4, pp. 681-693, 2003.

[11] K. W. Fong, A. Toi, S. Salem et al., "Detection of fetal structural abnormalities with US during early pregnancy," Radiographics, vol. 24, no. 1, pp. 157-174, 2004.

[12] O. Oztekin, D. Oztekin, S. Tinar, and Z. Adıbelli, "Ultrasonographic diagnosis of fetal structural abnormalities in prenatal screening at 11-14 weeks," Diagnostic and Interventional Radiology, vol. 15, pp. 221-225, 2009.

[13] A. C. Rossi and F. Prefumo, "Accuracy of ultrasonography at 1114 weeks of gestation for detection of fetal structural anomalies: a systematic review," Obstetrics and Gynecology, vol. 122, no. 6, pp. 1160-1167, 2013.

[14] D. Iliescu, S. Tudorache, A. Comanescu et al., "Improved detection rate of structural abnormalities in the first trimester using an extended examination protocol," Ultrasound in Obstetrics and Gynecology, vol. 42, no. 3, pp. 300-309, 2013.

[15] B. J. Whitlow, I. K. Chatzipapas, M. L. Lazanakis, R. A. Kadir, and D. L. Economides, "The value of sonography in early pregnancy for the detection of fetal abnormalities in an unselected population," British Journal of Obstetrics and Gynaecology, vol. 106, no. 9, pp. 929-936, 1999.

[16] L. A. Dolkart and F. T. Reimers, "Transvaginal fetal echocardiography in early pregnancy: normative data," American Journal of Obstetrics and Gynecology, vol. 165, no. 3, pp. 688-691, 1991.
[17] S. Saltvedt, H. Almström, M. Kublickas, L. Valentin, and C. Grunewald, "Detection of malformations in chromosomally normal fetuses by routine ultrasound at 12 or 18 weeks of gestation-a randomised controlled trial in 39572 pregnancies," BJOG, vol. 113, no. 6, pp. 664-674, 2006.

[18] M. A. Rustico, A. Benettoni, G. D’Ottavio et al., "Early screening for fetal cardiac anomalies by transvaginal echocardiography in an unselected population: the role of operator experience," Ultrasound in Obstetrics and Gynecology, vol. 16, no. 7, pp. 614$619,2000$. 


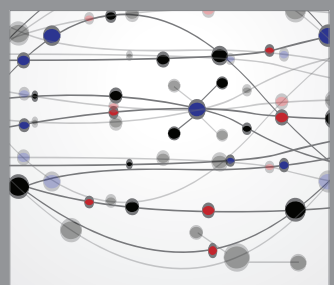

The Scientific World Journal
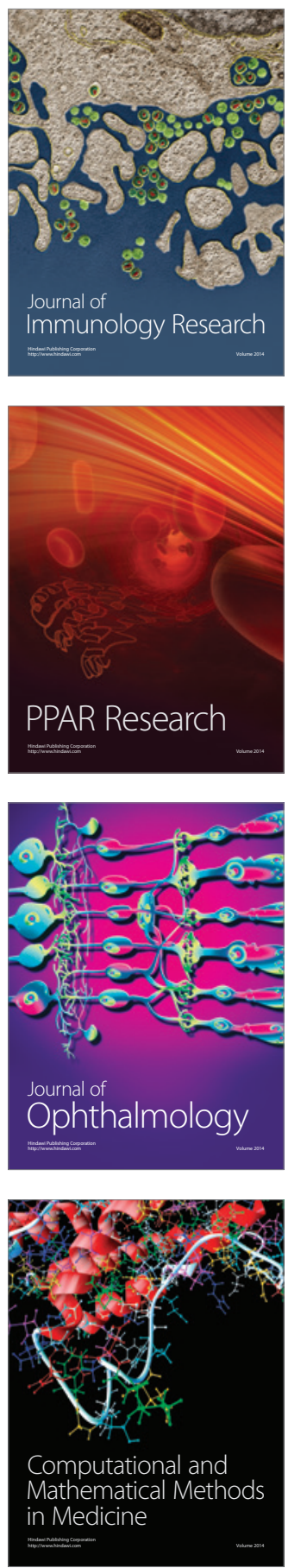

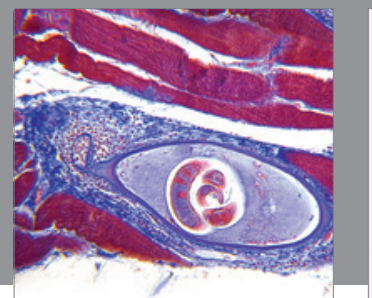

Gastroenterology

Research and Practice
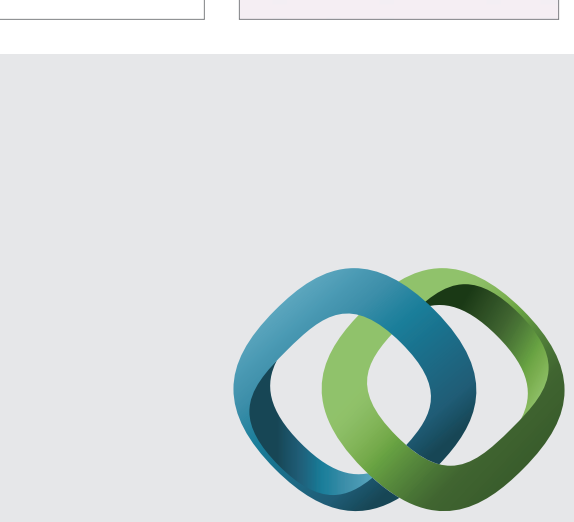

\section{Hindawi}

Submit your manuscripts at

http://www.hindawi.com
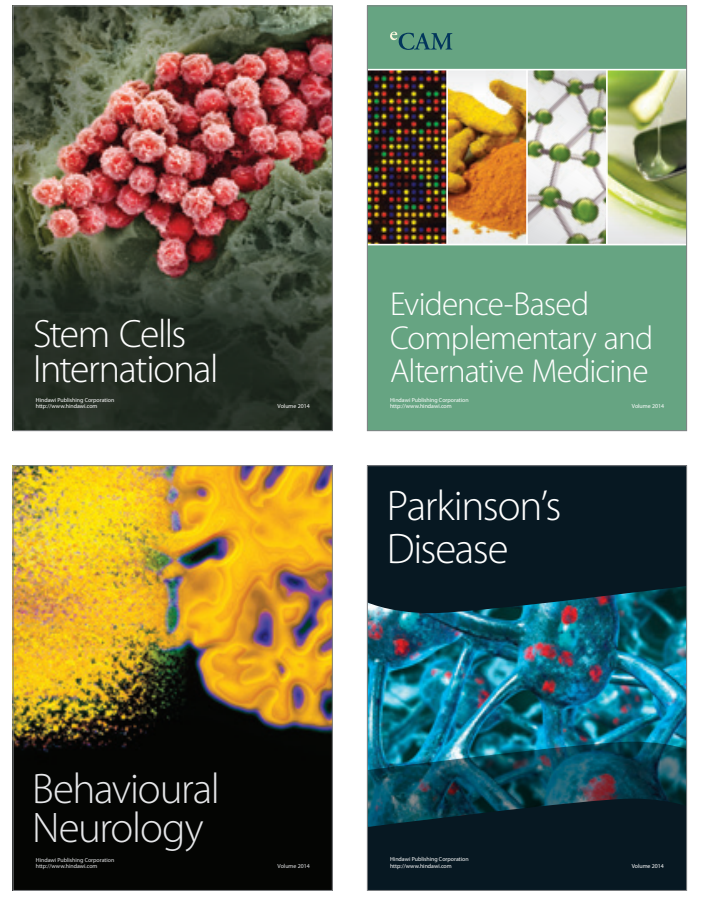
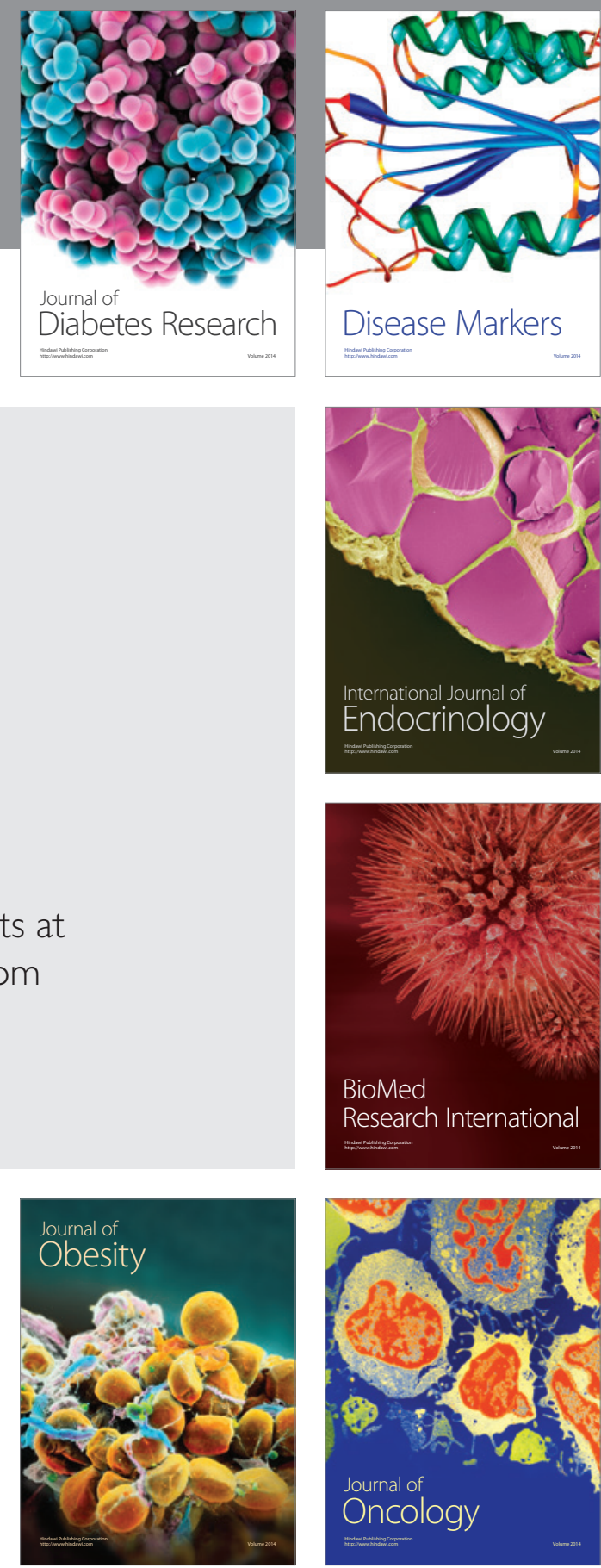

Disease Markers
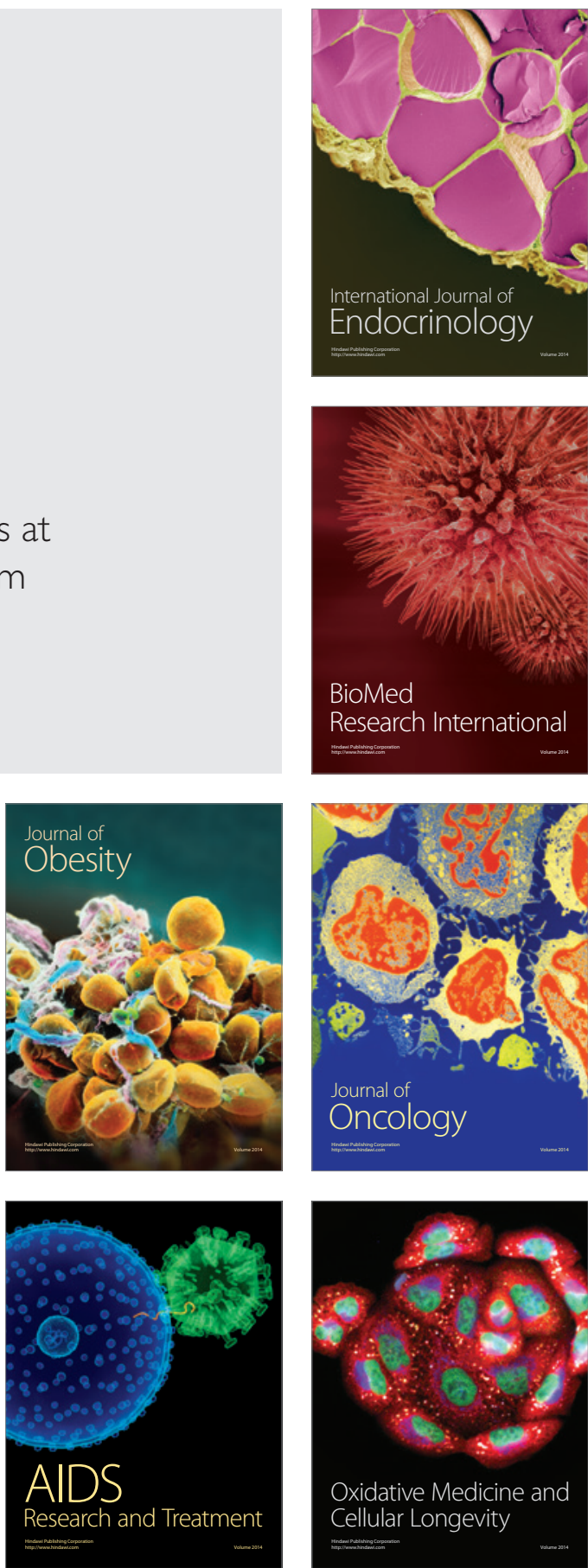